\title{
The cerebral hemispheres differ in their capacity for content-to-level binding but not for identification: Evidence from conjunction errors obtained with bilateral hierarchical stimuli
}

\author{
Rana Kruse and Ronald Hübner \\ Department of Psychology, Universität Konstanz, Konstanz, Germany
}

It is widely assumed that the right and left cerebral hemispheres are specialised for processing the global and local information of hierarchical stimuli, respectively. This idea has further been specified in the content-to-level binding theory (Hübner \& Volberg, 2005) by stating that the hemispheres differ in their efficiency for binding the contents of a stimulus to their respective level. In contrast, it is assumed that the hemispheres do not differ in their capacity for the identification of the information at the two levels. This latter hypothesis was tested in the present experiment by presenting a hierarchical letter to each visual field. As expected, there were visual field effects only for errors involving the erroneous binding between a letter and a level. For errors that result from the mislocalisation of a letter, there were no visual field effects. Together, the data support the hypothesis that the hemispheres do not differ in their identification capacity.

Keywords: Feature binding; Conjunction errors; Global/local processing; Visual field effects.

It is generally assumed that the left cerebral hemisphere (LH) preferentially processes local stimulus features, whereas the right hemisphere $(\mathrm{RH})$ is better prepared for the processing of global features. In fact, if we consider the relevant literature then we not only find that such hemispheric asymmetries have been observed in numerous electrophysiological studies (e.g., Han, Fan, Chen, \& Zhuo, 1999; Heinze \& Münte, 1993; Malinowski, Hübner, Keil, \& Gruber, 2002; Volberg \& Hübner, 2004), neuropsychological studies (e.g., Delis, Robertson, \& Efron, 1986; Robertson, Lamb, \& Knight, 1991), and brain-imaging studies (e.g., Fink et al., 1996; Heinze, Hinrichs, Scholz,

Address correspondence to: Ronald Hübner, Universität Konstanz, Fachbereich Psychologie, Fach D29, D-78457 Konstanz, Germany. E-mail: ronald.huebner@uni-konstanz.de 
Burchert, \& Mangun, 1998), but also that corresponding visual field (VF) effects showed up in behavioural studies with lateralised stimuli (e.g., Hübner \& Malinowski, 2002; Hübner, Volberg, \& Studer, 2007; Malinowski et al., 2002; Van Kleeck, 1989; Volberg \& Hübner, 2004; Yovel, Yovel, \& Levy, 2001). In most of these studies hierarchical letters (Navon, 1977) were applied as stimuli; i.e., large global letters constructed from copies of small local letters (see Figure 1).

Originally it was thought that the $\mathrm{LH}$ and $\mathrm{RH}$ constitute information channels or pathways specialised for the processing and transmission of local and global stimulus features, respectively, and that this separation of processing leads to a unique relation between the contents of a stimulus and their respective level (for overviews, see Van Kleeck, 1989; Yovel et al., 2001). However, Hübner and his co-workers (e.g., Hübner \& Malinowski, 2002; Hübner \& Volberg, 2005) have questioned that global/local processing proceeds this way. Rather, in their "content-to-level binding" (CLB) theory they proposed that there are at least two qualitatively different stages of processing: an early stage, where the contents of the levels of a hierarchical stimulus are identified independently of their level, and a later stage, where the identities are linked to their respective level. Moreover, it is assumed that the hemispheres do not differ in their identification capacity, but only in their efficiency to bind levels and identities: The RH preferentially binds the global level to its content, whereas the LH does this for the local level. We

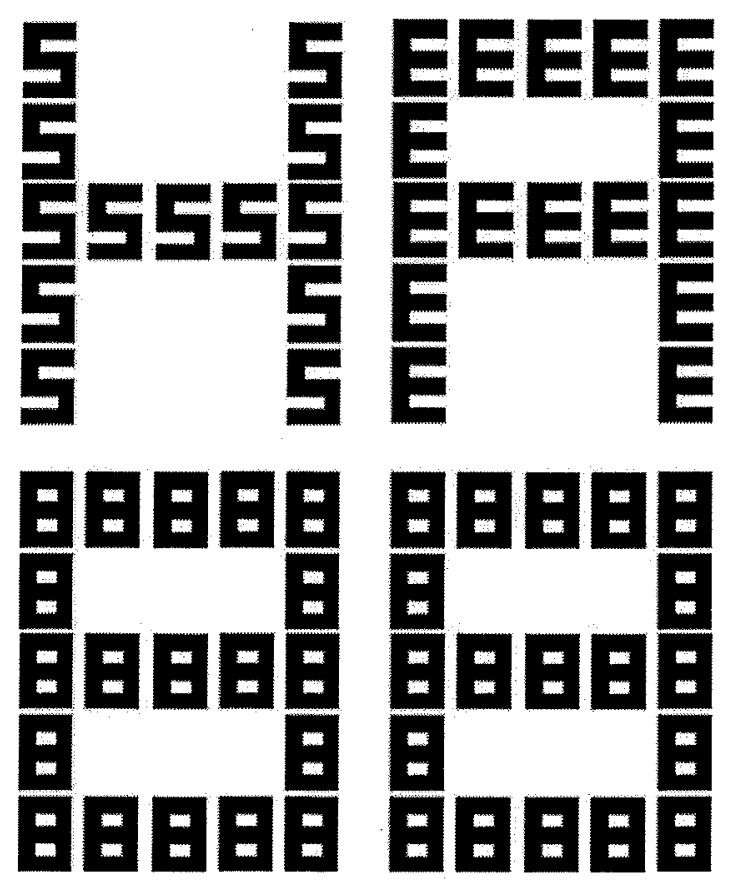

Figure 1. An example of the applied bilateral stimuli (top) and the corresponding mask (bottom). In the experiment the stimuli were presented in white on a black background. 
will call this specific assumption of the CLB theory the asymmetric-binding hypothesis.

Strong support for the CLB theory stems from experiments (e.g., Hübner \& Volberg, 2005) in which participants had to indicate the identity of the letter at a pre-specified target level of a laterally presented hierarchical letter. In these experiments the stimulus was masked shortly after its presentation. It was assumed that, if the CLB theory is right, then the masking should disturb the binding process, which in turn should produce conjunction errors between levels and identities (cf. Treisman \& Gelade, 1980). Consequently the participants should frequently report the letter at the non-target level. Indeed, this is exactly what has been observed (Hübner \& Volberg, 2005). Moreover, as predicted, there were also substantial VF effects. For the local level, more conjunction errors occurred for stimuli presented in the left visual field (LVF) than for those shown in the right visual field (RVF), whereas the opposite held for the global target level.

These results clearly show that the contents of a hierarchical stimulus must actively be bound to their respective level during the construction of a mental representation. However, up to now, little is known about the process of content-to-level binding. A problem with investigating the details of this process is that binding presupposes identification so that any observable rate of conjunction errors depends on both processes. For instance, the error rate increases if the binding efficiency decreases, but also if the identification rate decreases. Thus, if one varies some factor and observes how it affects the rate of conjunction errors, then one cannot decide whether identification or binding was affected, or even both.

A practical solution to this problem would be available if the asymmetricbinding hypothesis were valid. In this case the properties of the binding process could simply be investigated by observing VF effects. If the hemispheres do not differ in their identification capacity, then modulations of the VF effects can uniquely be attributed to variations of the binding efficiency. Indeed this approach has recently been applied by Flevaris, Bentin, and Robertson (2010). They investigated whether content-to-level binding can be improved by spatial-frequency priming. Their idea was that, if the priming of a target level improves the corresponding binding process, and if the inferior cerebral hemisphere for that level benefits more from the priming than the superior hemisphere (ceiling effect), then the respective $\mathrm{VF}$ effect should be reduced. In contrast, if priming merely improves identification, then the rate of conjunction errors should be reduced equally for each hemisphere, and consequently there should be no modulation of the VF effects. Flevaris et al. (2010) found, as expected, that priming with low and high spatial frequencies reduced the VF effects for the global and local level, respectively, and they concluded that spatial-frequency processing is related to the content-to-level binding. 
This is an important result. However, as mentioned, the rationale of Flevaris et al.'s (2010) experiment strongly depends on the validity of the asymmetric-binding hypothesis. Unfortunately, up to now, the support for this hypothesis has been weak. There is some evidence from response-time studies (e.g., Hübner et al., 2007; Schlösser, Hübner, \& Studer, 2009; Volberg \& Hübner, 2006) and electrophysiological studies (e.g., Malinowski et al., 2002; Volberg \& Hübner, 2004) demonstrating that hemispheric asymmetries occur more reliably with incongruent stimuli-i.e., stimuli whose content at the nontarget level activates the wrong response - than with congruent stimuli. Such a result is what one would expect if the asymmetric-binding hypothesis is correct, because hemispheric asymmetries should occur only in conditions in which binding is necessary. This holds for incongruent stimuli, as they produce a response conflict that can only be resolved by binding the letters to their respective level. In contrast, congruent stimuli exclusively activate the correct response, so that the information at the early stage is sufficient for response selection and allows participants to bypass the integration stage. This reasoning nicely accounts for the result that there are no hemispheric asymmetries with congruent stimuli.

Also in line with the asymmetric-binding hypothesis are experiments in which the interaction between VF and level was absent for non-conjunction errors (Flevaris et al., 2010; Experiment 1 in Hübner \& Volberg, 2005). However, this was not always the case. In some conditions this interaction was significant (e.g., Experiment 2, and one condition of Experiment 3 in Hübner \& Volberg, 2005). More direct evidence in favour of the asymmetricbinding hypothesis has been provided by the results of one condition in one of Hübner and Volberg's (2005) experiments, in which a task-irrelevant symbol was present at the non-target level, so that a letter was present only at the target level. Consequently, no conjunction errors were possible. As a result, no VF effects occurred in this condition, which indicates that letter identification was equally efficient in both hemispheres.

Thus, altogether, the asymmetric-binding hypothesis is supported by some indirect evidence, mainly from response time studies, and by the result of a single condition in a single experiment in Hübner and Volberg (2005). Given the importance of this hypothesis for investigating the role of content-tolevel binding for global/local processing, it seems necessary to provide more supporting results, which was the aim of the present study. Our idea was to apply the masking paradigm to a task in which not only binding between content and level is important, but also binding between content and location (visual field). When the hemispheres differ in their efficiency for content-to-level binding, but neither in their capacity for identification, nor in their efficiency for binding content and location, then VF effects should occur only for errors that result from failures to bind content to level. 
For our objective we used bilateral stimuli, i.e., we presented one hierarchical letter to the LVF and another to the RVF (see Figure 1). All four possible location/level combinations contained a different letter, i.e., there were four different letter identities in each display. Moreover the stimuli were masked after a variable stimulus-mask interval (SMI). Whereas the target level was pre-cued, the relevant location (target VF) was not specified before masking. Thus the task of the participants was to identify the letter at the pre-specified target level of the stimulus in the post-specified target VF. If they failed to see a letter at the target position and level and also the other letters then, by instruction, they should guess the identity from the four alternatives. In case some of the other letters are seen at non-target locations and levels, they should guess the identity from the remaining alternatives. With our experimental design all possible errors are conjunction errors by definition. However, they can be categorised into three different types. First, participants could make level errors, i.e., falsely state the letter at the non-target level of the target stimulus. Second, they could make location errors, i.e., falsely report the letter at the target level of the non-target stimulus, i.e., the stimulus in the opposite VF. Finally, they could make level-location errors by stating the letter at the non-target level of the stimulus in the opposite VF. Because guessing was possible, we had to take its possible contribution into account. If we assume the worst case, that no letter is identified on all error trials, then all error responses would originate from pure guessing. In this case all error types would have the same proportion of $1 / 3$ of the total error rate. Thus, if we observe proportions that differ from $1 / 3$, then we can be sure that letters had been identified but bound to a wrong level or location. Moreover, pure guessing would predict that there should be no VF effects.

With this procedure we tested the asymmetric-binding hypothesis, i.e., the assumption that the hemispheres differ only in their efficiency for content-tolevel binding, but not with respect to identification. If this hypothesis is correct, then VF effects should occur only for conjunction errors across levels, but not for those across locations.

\section{METHOD}

\section{Participants}

A total of 28 students (mean age of 22 years; 4 male) from the Universität Konstanz, Germany, participated in the experiment. All had normal or corrected-to-normal vision, were right-handed by self-report, and were paid $€ 8$ for their participation. 


\section{Apparatus and stimuli}

Stimuli were presented on an 18-inch colour monitor with a resolution of $1280 \times 1024$ pixels and a refresh rate of $60 \mathrm{~Hz}$. Participants responded by pressing one of four buttons of a computer keyboard. Stimulus presentation as well as response registration was controlled by the same personal computer (PC).

Each stimulus display consisted of two hierarchical letters (for examples see Figure 1). One of the two hierarchical letters was constructed from two out of four different letters $(A, S, H, E)$, and the other from the two remaining letters. The size of the global letters was $4.48^{\circ}$ of visual angle horizontally and $5.72^{\circ}$ vertically. The respective size of the local letters was $0.72^{\circ} \times 1.08^{\circ}$. Stimuli were presented in white on a black background to the LVF as well as to the RVF at an eccentricity of $2.82^{\circ}$ (from the midline of the screen to the centre of the stimulus).

\section{Procedure}

Participants were seated at a viewing distance of approximately $60 \mathrm{~cm}$ in front of the screen. A trial started with the presentation of a cue (the letter 1 or $g$ to indicate a local or global target level, respectively) at the centre of the screen for $300 \mathrm{~ms}$, followed by a fixation cross for $300 \mathrm{~ms}$. Then the stimulus was presented for $32 \mathrm{~ms}$. After a stimulus-mask interval (SMI) of 32, 64, or $96 \mathrm{~ms}$ the mask (see Figure 1) was presented. Simultaneous with the mask a post-cue appeared, which was located below the mask, and which consisted of the word "links" or "rechts" (the German words for "left" or "right") to indicate the target location. The mask remained on the screen until the response. VF, target level, and SMI were randomised across trials. The task of the participants was to identify the letter at the pre-cued level of the postcued stimulus. Participants responded by pushing one of four response buttons of the keyboard (each corresponding to a certain letter). The buttonto-letter mapping was varied across participants to counteract any response biases. Participants were advised to respond without time pressure, and that each stimulus display contained all four different letters. They were further told that, should they identify the letter at the non-target level or letters of the non-target stimulus, they should not report these letters but guess from the remaining ones. At the end of each block a feedback screen was displayed informing the participants about their error rate on the current block. If the error rate exceeded $50 \%$ the participants were asked to increase their effort.

After some training (four blocks of 24 trials with a decreasing SMI; 192 $\mathrm{ms}, 96 \mathrm{~ms}$, and $64 \mathrm{~ms}$ ), eight blocks of 72 trials were run for each participant in a single 1-hour session. This resulted in 48 trials per condition. 


\section{RESULTS}

\section{Overall analyses}

The overall mean error rate was $34.9 \%$. In a first analysis the error rates, as shown in Figure 2, were entered into a three-factor ANOVA on the withinparticipants factors target level (global, or local), visual field (LVF, or RVF), and SMI (short, medium, or long).The main effects of target level, $F(1,27)=4.87, p<.05$, visual field, $F(1,27)=41.7, p<.001$, and $S M I$, $F(2,54)=102, p<.001$, were significant. More errors occurred for the local target level than for the global one (38.3\% versus $31.5 \%$ ), and more errors were made for RVF targets than for LVF targets (45.8\% versus $24.0 \%$ ). Furthermore, the rate of errors decreased with an increasing SMI $(41.7 \%$, $33.8 \%, 29.2 \%$ ). There was also a significant two-way interaction between target level and visual field, $F(1,27)=17.6, p<.001$, reflecting that for LVF targets more errors occurred for the local level than for the global level $(31.7 \%$ versus $16.4 \%)$, whereas there was a small trend in opposite direction for RVF targets $(45.0 \%$ versus $46.6 \%)$. The two-way interaction between visual field and $S M I$ was also significant, $F(2,57)=8.70, p<.001$. It indicates that for LVF targets the error rate decreased to a larger extent from the long SMI to the medium SMI than from the medium SMI to the short SMI, whereas for RVF targets the decrease between the SMIs was of similar size. Finally, the three-way interaction between target level, visual field, and $S M I$ was significant, $F(2,57)=7.72, p<.01$. It indicates that the two-way interaction between visual field and $S M I$ was more pronounced for the local level (LVF: $42.0 \%, 28.6 \%, 24.4 \%$; RVF: 48.7\%, 46.4\%, 39.9\%) than for the global one (LVF: 22.6\%, 15.0\%, 11.6\%; RVF: 53.7\%, 45.2\%, 41.0\%).

To test whether there was a difference between the three error types we ran the same analysis as before, but this time also included the factor error type

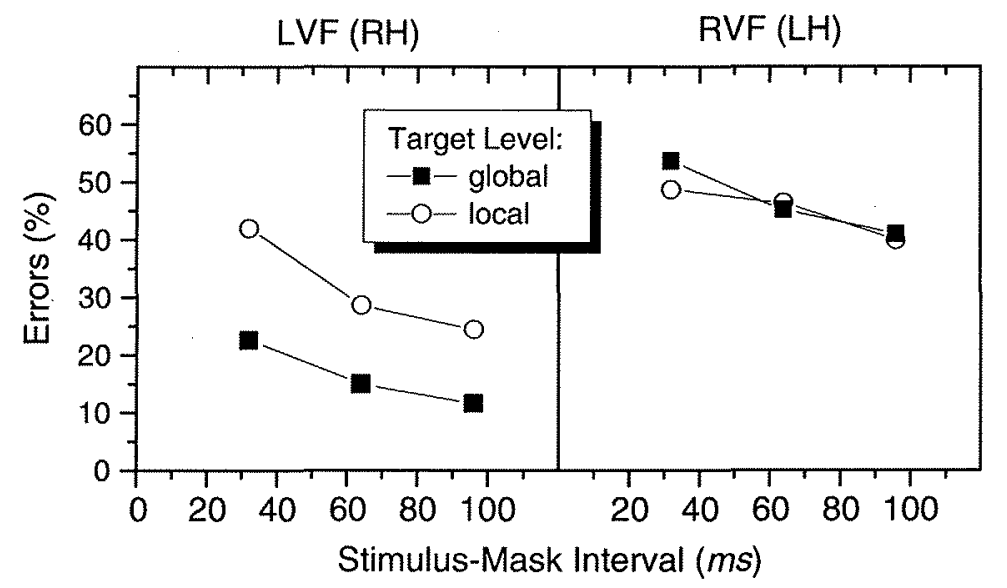

Figure 2. Error rates for the local and global target level and the two visual fields. 
(level, location, or level-location), which turned out to be highly significant, $F(2,54)=30.0, p<.001$. Most errors were level errors $(16.5 \%)$, least errors were location errors $(7.35 \%)$, and the level-location errors were in between $(10.7 \%)$. Planned comparisons revealed that all pair-wise differences were significant. Moreover, the ANOVA also showed that there was a significant three-way interaction between the factors error-type, level, and visual field, $F(2,54)=13.6, p<.001$, whereas the four-way interaction between all factors was far from significance, $F(4,108)=0.748, p=.561$. To examine the three-way interaction in more detail, the rates for the different error types were analysed in separate three-factor ANOVAs on the within-participants factors target level (global, local), visual field (LVF, RVF), and SMI (short, medium or long). The data for the different error types and conditions are shown in Figure 3.

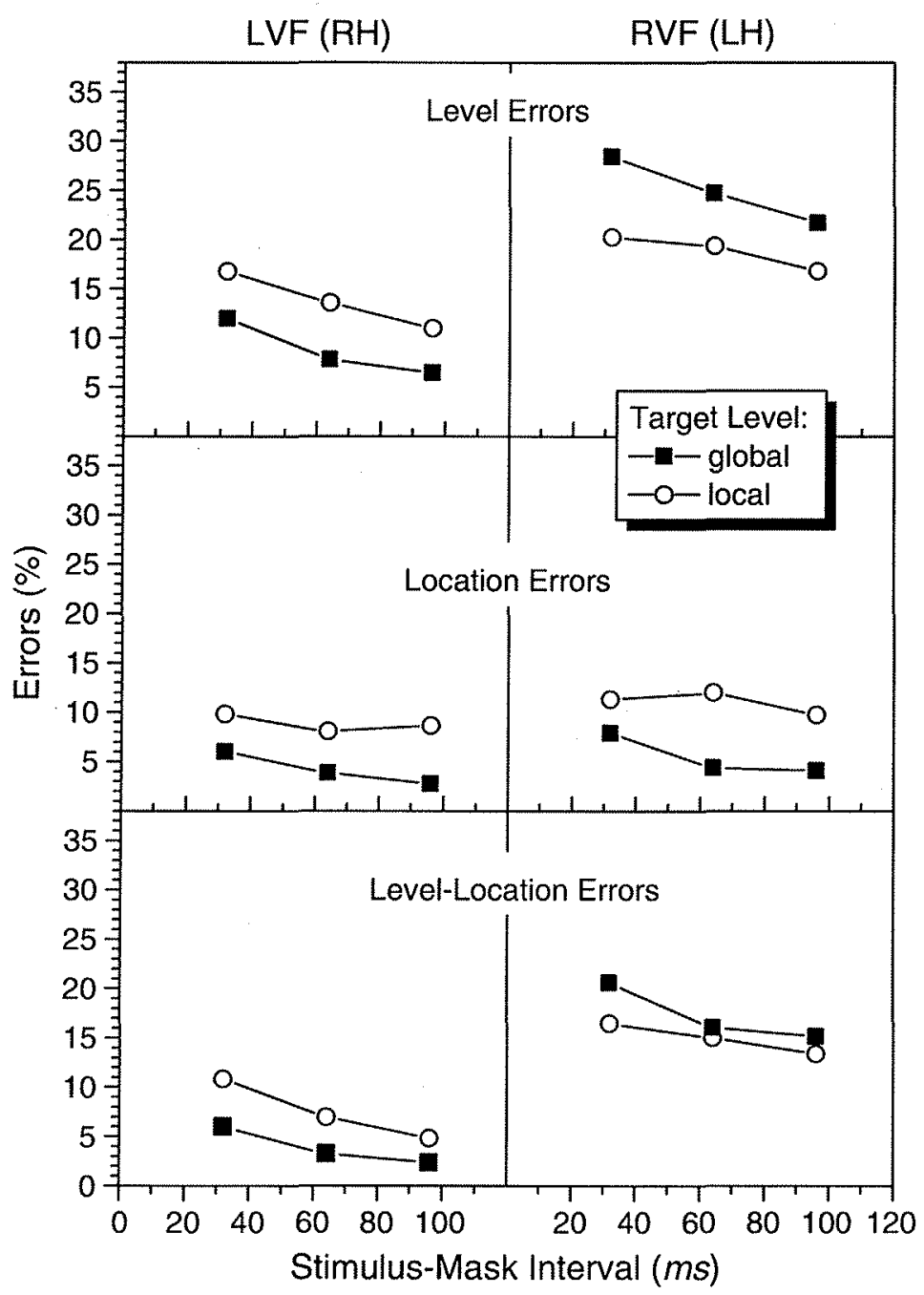

Figure 3. Error rates separated for the different types. 


\section{Level errors}

Level errors resulted from erroneously indicating the letter at the non-target level of the target stimulus, i.e., of the stimulus in the target visual field. The mean rate of level errors was $16.5 \%$, which was significantly larger, $F(1$, $27)=42.1, p<.001$, than the rate of $11.6 \%$, which would be expected from pure guessing.

The main effect of visual field was significant, $F(1,27)=54.1, p<.001$. There were more level errors for stimuli in the LVF than for those in the RVF $(11.2 \%$ versus $21.7 \%)$. However, visual field interacted significantly with target level, $F(1,27)=18.0, p<.001$. For LVF targets more errors occurred for local targets than for global ones $(8.49 \%$ versus $13.9 \%)$, whereas the opposite held for RVF targets (24.7\% versus $18.8 \%$ ). Planned comparisons revealed that the difference was significant for LVF targets, $F(1,27)=12.6$, $p<.01$, as well as for RVF targets, $F(1,27)=6.98, p<.05$. The main effect of $S M I$ was also significant, $F(2,54)=35.4, p<.001$. As can be seen in Figure 3 , the error rate decreased $(19.1 \%, 16.4 \%, 14.0 \%)$ with an increasing SMI.

\section{Location errors}

Location errors resulted from erroneously indicating the letter at the target level of non-target stimulus, i.e., of the stimulus in the opposite visual field. The mean rate of this error type was $7.35 \%$, which was significantly smaller, $F(1,27)=14.5, p<.001$, than the rate of $11.6 \%$ predicted by pure guessing.

The main effect of target level was significant, $F(1,27)=8.15, p<.01$, indicating that more location errors occurred for the local target level than for the global target level $(10.1 \%$ versus $4.56 \%)$. Also the main effect of visual field was significant, $F(1,24)=9.01, p<.01$. More errors occurred for RVFtargets than for LVF-targets ( $8.16 \%$ versus $6.54 \%$ ). Finally, the main effect of $S M I$ was significant, $F(2,54)=7.65, p<.01$, indicating that location errors decreased with an increasing SMI.

\section{Level-location errors}

This error type resulted from erroneously indicating the letter at the nontarget level of non-target stimulus, i.e., of the stimulus in the non-target visual field. The mean rate of level-location errors was $10.7 \%$, which was not significantly different, $F(1,27)=1.51, p=.229$, from the pure guessing rate of $11.6 \%$.

The main effects of visual field, $F(1,27)=42.3, p<.001$, and of $S M I$, $F(2,54)=27.4, p<.001$, were significant. They indicate that more levellocation errors occurred for RVF-targets than for LVF-targets (15.9\% versus $5.52 \%$ ), and that the errors decreased with an increasing SMI. There was also a two-way interaction between visual field and target level, $F(1,27)=7.55$, 
$\mathrm{p}<.05$. It indicates that for LVF targets more errors occurred for the local level than for the global level $(7.25 \%$ versus $3.80 \%)$, whereas the opposite held for RVF targets (14.9\% versus $16.9 \%$ ). Planned comparisons showed that the difference was significant only for LVF stimuli, $F(1,27)=8.40$, $p<.01$.

A further comparison between this error type and the level errors revealed that the visual field effects were significantly smaller for the level-location errors, $F(1,27)=5.31, p<.05$.

\section{DISCUSSION}

The objective of this study was to test the asymmetric-binding hypothesis, which is an important part of the CLB theory (Hübner \& Volberg, 2005) and states that the cerebral hemispheres differ with respect to the content-to-level binding but not in their capacity for identification. This hypothesis is not only important by itself, but also because it allows one to use VF effects for investigating the mechanisms of content-to-level binding, as has impressively been demonstrated by Flevaris et al. (2010). To test the hypothesis we simultaneously presented one hierarchical letter to the LVF and another to the RVF. The stimuli were masked after one of three short stimulus-mask intervals (SMIs), and the task of the participants was to indicate the letter at a pre-specified target level of the stimulus in the post-specified VF.

The overall results show that the error rates decreased with an increasing SMI, and that they varied with target level and VF. Moreover, there was a general LVF-advantage that held for both target levels. Presumably, because the target location was cued only after the stimuli had disappeared, the participants tried to encode the information from both stimuli by attentionally scanning the stimuli in the reading direction, i.e., from left to right. Interestingly, the LVF stimulus profited more from an increased SMI than the RVF stimulus, at least with respect to the increase from the shortest SMI to the medium SMI (see Figure 2). This could mean that the participants put generally more emphasis on LVF stimuli, or that they switched attention forth and back between the stimuli. With respect to the latter strategy it might then have been possible on trials with a medium SMI to return attention to the LVF stimulus but not to move attention again to the RVF stimulus, which could explain the relatively large improvement for that condition. Presumably, however, there was a mixture of different strategies. Alternatively, it is also possible that the LVF advantage reflects the RH superiority of attentional orienting (e.g., Becker \& Karnath, 2007; Bowen, McKenna, \& Tallis, 1999; Shulman, d'Avossa, Tansy, \& Corbetta, 2002).

As can also be seen in Figure 2, the general LVF advantage had the effect that the expected hemispheric differences were expressed by a larger LVF 
advantage for the global level than for the local level. By subtracting the average LVF advantage from the data, one would observe the usual VF effects, i.e., a LVF advantage for the global level and a RVF advantage for the local level. However, instead of removing the average LVF advantage from the data, we can simply compare the errors for the target levels within a given VF. This makes sense especially if we consider the individual error types.

Because all employed letter identities were present in each stimulus display, three specific error types were possible: level errors, location errors, and level-location errors. The overall error rate was about 35\%, which indicates that the mask effectively interrupted stimulus processing. If the masking had completely prevented letter identification or binding, then pure guessing would have been the only strategy to select a response on error trials. In this case each error type should have a proportion of about $11.6 \%$. As our analyses revealed, though, the rates differed substantially between the different error types (see Figure 3). This demonstrates that errors were not only due to guessing.

First of all, the proportion of level errors was significantly greater than $1 / 3$, which indicates that there were real conjunction errors. That is, when target identification failed, then the letter at the non-target level of the target stimulus was sometimes falsely bound to the target level. In other words, the letters sometimes migrated from one level to the other within a given stimulus. These migrations occurred less often in LVF stimuli than in RVF stimuli, which was presumably the result of the left-to-right scanning strategy. However, there was also a significant interaction between VF and level. As can be seen in Figure 3, LVF stimuli produced fewer conjunction errors for the global target level than for the local target level, whereas the opposite held for RVF stimuli. These results nicely reflect the proposed hemispheric asymmetries for global/local processing (Hübner \& Volberg, 2005; Robertson \& Ivry, 2000). Thus, taken together, the high rate of level errors and the VF effects strongly indicate that there were real conjunction errors between the levels and letters within a hierarchical stimulus, i.e., that the letters migrated between the two levels.

Compared to the level errors, there were fewer location errors. Their proportion was significantly smaller than $1 / 3$ of all errors, which indicates that letter-to-location binding was relatively successful, at least for the target level. When the letter at the target level in the target VF had not been identified correctly, then it was highly likely that the letter at the target level of the stimulus in the non-target VF had been identified and bound correctly to its location. Consequently, this letter could be excluded as a potential response. Note that binding this letter to its level was not necessary for its exclusion.

It is surprising that letter-to-location binding was so successful, despite the fact that the participants did not know the target location in advance. 
Perhaps the left-to-right scanning strategy was a helpful strategy in this respect. Interestingly, although significant, the main effect of VF was much smaller than that for the other error types (see Figure 3). Moreover, this effect was not modulated by the SMI. Together, these results suggest that a one-pass scanning from left to right was often sufficient for identifying the two letters at the target level and for binding them to their location (but not necessarily to their level).

Most important for the objective of the present study, there was no interaction between target level and VF for the location errors. This shows that, when location instead of level can be used to exclude letters as possible responses, then the cerebral hemispheres do not differ in their performance. Crucially this also implies that their capacity is similar with respect to the identification of global and local letters, which confirms the asymmetricbinding hypothesis.

If we finally consider the rate of the level-location errors, then we see that it was not significantly different from $1 / 3$ of the overall error rate. Because such a proportion could have originated from pure guessing, we cannot be sure that the level-location errors also include real conjunction errors. What speaks against a pure guessing account is the fact that there was a significant interaction between target level and VF, even though this interaction was substantially weaker than that for the level errors. Thus, although the evidence is relatively weak for the assumption that the letter from the nontarget level of the stimulus in the opposite visual field was falsely bound to the target level of the target stimulus, it is nevertheless not unlikely that such conjunction errors occurred.

Altogether, our data strongly support the asymmetric-binding hypothesis. In cases where erroneous bindings between levels and identities occurred, the hemispheres differed significantly. In contrast, for mislocations of letter identities at the same level there were no VF effects. This demonstrates that the RH and LH are specialised only for the binding of identities to the global and local level of a hierarchical stimulus, respectively. They are equally efficient, though, with respect to identification, irrespective of the level at which an item occurs. Given this indifference we can consider variations of VF effects for conjunction errors as exclusively indicating modulations of the content-to-level binding processes, which provides a useful procedure for investigating these binding processes, as has already been demonstrated by Flevaris et al. (2010). The present results increase our confidence that this procedure is valid. 


\section{REFERENCES}

Becker, E., \& Karnath, H. O. (2007). Incidence of visual extinction after left versus right hemisphere stroke. Stroke, 38, 3172-3174.

Bowen, A., McKenna, K., \& Tallis, R. C. (1999). Reasons for variability in the reported rate of occurrence of unilateral spatial neglect after stroke. Stroke, 30, 1196-1202.

Delis, D. C., Robertson, L. C., \& Efron, R. (1986). Hemispheric specialisation of memory for visual hierarchical stimuli. Neuropsychologia, 24, 205-214.

Fink, G. R., Halligan, P. W., Marshall, J. C., Frith, C. D., Frackowiak, R. S. J., \& Dolan, R. J. (1996). Where in the brain does visual attention select the forest and the trees? Nature, 382, 626-628.

Flevaris, A. V., Bentin, S., \& Robertson, L. C. (2010). Local or global?: Attentional selection of spatial frequencies binds shapes to hierarchical levels. Psychological Science, 2I, 424-431.

Han, S., Fan, S., Chen, L., \& Zhuo, Y. (1999). Modulation of brain activities by hierarchical processing: A high-density ERP study. Brain Topography, 11, 171-183.

Heinze, H. J., Hinrichs, H., Scholz, M., Burchert, W., \& Mangun, G. R. (1998). Neural mechanisms of global and local processing: A combined PET and ERP study. Journal of Cognitive Neuroscience, 10, 485-498.

Heinze, H. J., \& Münte, T. F. (1993). Electrophysiological correlates of hierarchical stimulus processing: Dissociation between onset and later stages of global and local target processing. Neuropsychologia, 31, 841-852.

Hübner, R., \& Malinowski, P. (2002). The effect of response competition on functional hemispheric asymmetries for global/local processing. Perception \& Psychophysics, 64, 1290-1300.

Hübner, R., \& Volberg, G. (2005). The integration of object levels and their content: A theory of global/local processing and related hemispheric differences. Journal of Experimental Psychology: Human Perception and Performance, 31, 520-541.

Hübner, R., Volberg, G., \& Studer, T. (2007). Hemispheric differences for global/local processing in divided attention tasks: Further evidence for the integration theory. Perception \& Psychophysics, 413-421.

Malinowski, P., Hübner, R., Keil, A., \& Gruber, T. (2002). The influence of response competition on cerebral asymmetries for processing hierarchical stimuli revealed by ERP recordings. Experimental Brain Research, 144, 136-139.

Navon, D. (1977). Forest before the trees: The precedence of global features in visual perception. Cognitive Psychology, 9, 353-393.

Robertson, L. C., \& Ivry, R. (2000). Hemispheric asymmetries: Attention to visual and auditory primitives. Current Directions in Psychological Science, 9, 59-63.

Robertson, L. C., Lamb, M. R., \& Knight, R. T. (1991). Normal global-local analysis in patients with dorsolateral frontal lobe lesions. Neuropsychologia, 29, 959-967.

Schlösser, J., Hübner, R., \& Studer, T. (2009). The effect of element spacing on hemispheric asymmetries for global/local processing. Experimental Psychology, 56, 321-328.

Shulman, G. L., d'Avossa, G., Tansy, A. P., \& Corbetta, M. (2002). Two attentional processes in the parietal lobe. Cerebral Cortex, 12, 1124-1131.

Treisman, A., \& Gelade, G. (1980). A feature-integration theory of attention. Cognitive Psychology, $12,97-136$.

Van Kleeck, M. H. (1989). Hemispheric differences in global versus local processing of hierarchical visual stimuli by normal subjects: New data and a meta-analysis of previous studies. Neuropsychologia, 27, 1165-1178.

Volberg, G., \& Hübner, R. (2004). On the role of response conflicts and stimulus position for hemispheric differences in global/local processing: An ERP study. Neuropsychologia, 42, $1805-1813$. 
Volberg, G., \& Hübner, R. (2006). Hemispheric differences for the integration of stimulus levels and their contents: Evidence from bilateral presentations. Perception \& Psychophysics, 68, 1274-1285.

Yovel, G., Yovel, I., \& Levy, J. (2001). Hemispheric asymmetries for global and local visual perception: Effects of stimulus and task factors. Journal of Experimental Psychology: Human Perception and Performance, 27, 1369-1385. 